\title{
Checklist for Phytodiversity of East Karbi Anglong Wildlife Sanctuary, Assam, Northeast India: Part I
}

\section{Puranjoy Mipun ${ }^{1 *}$, Dipankar Borah ${ }^{2}$, Nazir Ahmad Bhat ${ }^{1}$, Yogendra Kumar ${ }^{1}$}

'Centre for Advanced Studies in Botany, North Eastern Hill University, Shillong, Meghalaya, 793022, India

${ }^{2}$ Department of Botany, Rajiv Gandhi University, Rono Hills, Doimukh, Arunachal Pradesh, 791112, India

Key words: Angiosperms, Floral Diversity, Threatened species

Study area: Karbi Anglong, Assam

Coordinate: $24^{\circ} 33^{\prime} \mathrm{N}$ to $26^{\circ} 35^{\prime} \mathrm{N}$; and $92^{\circ} 10^{\prime} \mathrm{E}$ to $93^{\circ} 50^{\prime} \mathrm{E}$

\section{Introduction:}

Karbi Anglong district, the largest district of Assam is home to five Wildlife Sanctuaries viz., Nambor, East KarbiAnglong, North KarbiAnglong, Garampani, and Marat Longri Wildlife Sanctuary. East KarbiAnglong Wildlife sanctuary (EKAWLS) is situated in the eastern part of KarbiAnglong district and serves as an essential component of the KarbiAnglong-Kaziranga landscape, recognized as one of the rich floral and faunal diversity region within the Indo-Burma biodiversity hotspot (WWF, 2002). During recent decades due to several anthropogenic activities viz., over-exploitation, habitat destruction, and fragmentation of forest areas have substantially altered the natural landscapes affecting the distribution of species populations and habitats (Hansen et al., 2013), which simultaneously hinder the sufficient propagation of the plant in its natural condition (Borthakur et al., 2018).

Moreover, the indigenous flora of a region today is mostly conf ined to the native forests found in the protected areas such as National Parks, Biosphere Reserves, Wildlife Sanctuaries, etc. Protected areas along with conserving the biodiversity, it also serve as indispensable to forest dwellers for subsistence and livelihoods (Phangchopi et al., 2017). Even these protected areas are under tremendous anthropogenic pressure, and several floristic elements face the threat of extinction. So, knowing the phyto-diversity of protected areas with its threatened species status could serve as a baseline for future conservation. In spite of numerous steps taken for cataloging the biodiversity

\section{Abstract}

This work presents a checklist of plant species in East KarbiAnglong Wildlife Sanctuary (EKAWLS), Assam, India. Inventorization was carried out from 2015-2018, and a total of 252 plant species are recorded belonging to 203 genera and 87 families. Fabaceae ( 25 species) is the most dominant family, and Clerodendrum ( 4 species) is the most species-rich genera. The enumerated taxa are presented with their vernacular names, habit form, and voucher numbers. Threat status of each taxon according to IUCN Red List categories is also provided. Anthropogenic activities and other natural calamities are causing serious threats particularly to the threatened species of the sanctuary. Population survey of all the threatened species was done based on the number of seedling, sapling, and adult from the study site.

present in the protected areas, studies in Northeast, India is still lacking (Bora \& Bhattacharyya, 2017). Moreover, considering the increased threats from large- scale deforestation and degradation of the natural habitat during the recent decades (Ronghang et al., 2012), it is urgent to have floristic studies of these sanctuaries before the floristic elements are lost forever. Unfortunately, till date, no floristic studies have been published from any of the sanctuaries as mentioned above, apart from some relatable works by Sankar \& Chowdhury (2000) reporting 431 plant species having different ethnobotanical categories and Gogoi \& Yonzone (2013) on orchids of the district. Several workers have undertaken publications in aspect to small localities within the district focusing on the ethnobotany (Kar \& Borthakur, 2008; Teron \& Borthakur, 2009, 2012, 2013; Teronpi et al., 2015; Phongchopi et al., 2014). The present study is focused on providing a checklist of the Phytodiversity of East Karbi Anglong Wildlife Sanctuary and to survey the population status of the collected threatened species.

\section{Methodology:}

Study area: The present study was carried out in East Karbi Anglong Wildlife Sanctuary (EKAWLS) of Karbi Anglong district of Assam, which is one of the primary forests of the state covering an area of $221.81 \mathrm{~km}^{2}$. It is in the elevation ranges from 8o-6oom a.s.l. The Topography is varied with undulating hills, wide valleys, steep gorges, rivers and rivulets, annual and perennial streams. The wildlife

*Corresponding Author: mipunpuranjoy@gmail.com 
sanctuary holds different forest types ranging from moist semi-evergreen, moist mixed deciduous to miscellaneous type with scattered pure or mixed patches of bamboos. The soil is well-drained, sandy loamy to clayey loamy, and the climate is warm and humid with an annual rainfall of 1800 $\mathrm{mm}$. The average temperature is around $24^{\circ} \mathrm{C}$.

Field survey and data collection: extensive field studies were conducted from 2015-2018 covering almost all seasons, focusing mostly on the flowering and fruiting phases. The surveys were done after seeking permission from the Principal Chief Conservator of Forests, Karbi Anglong Division (O.O. No. 43/Memo No. Addl. PCCF/KA/Wildlife/2015-16/886-89, dated Diphu, 15 September 2015). The materials were processed according to the methodology followed by Jain \& Rao (1977), and depositions were made into NEHU herbaria. The species identifications were carried out by consulting specimens housed at regional herbaria (NEHU, ASSAM, ARUN), with images of type specimens available in online databases and specialized bibliography and specialists. The International Plant Name Index (2019) and The Plant List (2019) (http://www.ipni.org) were consulted for current nomenclature of the taxa. The resulting floristic list is consistent with Angiosperm Phylogeny Group IV (2016) system of classif ication and arranged in alphabetical order in both the family and generic level. The IUCN Red List (2019) (http://www.iucnredlist.org) was used to categorize species into different Red List categories. Population and regeneration studies of the threatened plants were done based on the density of seedling, sapling, and adult individuals within the study site following the methodology Mir et al., (2017 a,b)."

\section{Results and Discussion:}

A total of 252 species belonging to 203 genera and 87 families was collected (Table-1). The lifeform of the collected taxa was highest for herbs (37\%) followed by trees (34\%), shrubs (28\%) and climbers (1\%). Among the representing families, Fabaceae was the most dominant with a total of 25 species under it, followed by Poaceae (14), Malvaceae (10), Zingiberaceae, Rubiaceae, Orchidaceae and Lamiaceae with 9 species each, Phyllanthaceae (8), Araceae (7), Moraceae, CyperaceaeCompositae, Apocynaceae and Acanthaceae with 6 species each,
Rutaceae, Lythraceae, Euphorbiaceae and Araliaceae with 4 species each, Vitaceae, Solanaceae, Magnoliaceae, Balsaminaceae, Arecaceae, Apiaceae, Annonaceae and Amaranthaceae with 3 species each, Anacardiaceae, Calophyllaceae, Combretaceae, Commelinaceae, Convolvulaceae, Fagaceae, Gesneriaceae, Linderniaceae, Meliaceae, Menispermaceae, Musaceae, Actinidiaceae, Myrtaceae, Olacaceae, Oxalidaceae, Piperaceae, Polygonaceae, Sapindaceae, Simaroubaceae, Theaceae and Urticaceae with 2 species each and rest 40 families with 1 species each. Clerodendrum was the most species-rich genera with four species followed by Phlogacanthus, Impatiens, Cyperus, Dalbergia, Senna, Magnolia, Ficus, Phyllanthus and Bambusa (3 species each), 29 Genera with two species each and rest 164 genera with one species each.

The diversity of the Wildlife sanctuary is scattered due to the sporadic occurrence of bamboo patches over large areas. Bauhinia variegata L., Senna siamea (Lam.) Irwin \& Barneby, Erythrina stricta Roxb., Litsea monopetala (Roxb.) Pers. among the trees, Tabernaemontana divaricata (L.) R.Br. ex Roem. \& Schult, Dracaena angustifolia (Medik.) Roxb., Chloranthus elatior Link among the shrubs and Impatiens balsamina L., Drymaria cordata (L.) Willd. ex Schult., Bidens pilosa L. among the herbs were most commonly found and distributed throughout all the parts of the wildlife sanctuary. Geodorum densiflorum (Lam.) Schltr., Geophila repens (L.) Johnst., Habenaria dentata (Sw.) Schltr., Brucea mollis Wall. ex Kurz, Hydnocarpus kurzii (King) Warb., Caryota urens L. were locally rare within the forest with a very low number of occurrences. Most of the plants have been traditionally utilized by the communities living in the periphery such as Abrus precatorius L., Abroma augusta (L.) L. f., Stemona tuberosa Lour. are used for medicine, Chisocheton cumingianus (C.DC.) Harms, Magnolia hodgsonii (Hook \& Thomson) Keng, Magnolia insignis Wall., Engelhardia spicata Lesch. ex Blume for timber, Clinopodium umbrosum (M. Bieb.) Kuntze, Boesenbergia longiflora (Wall.) Kuntze, Zanthoxylum rhetsa (Roxb.) DC. as food items. Though the forest is mostly degraded in its outer parts, the core area is rich in terms of plant life with high endemism. Some endemic taxa of Northeast India present within the boundaries of the sanctuary are Larsenianthus careyanus (Benth.) Kress \& Mood, Larsenianthus assamensis Dey,

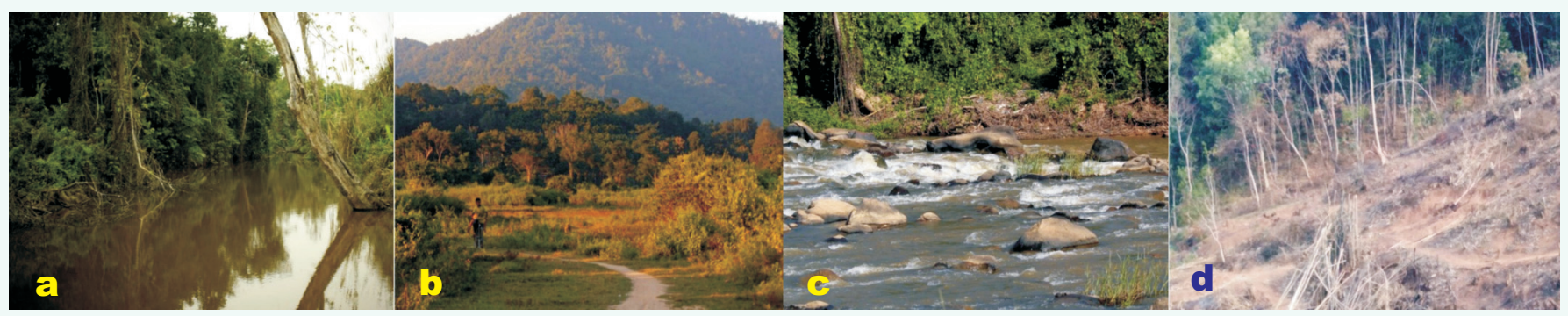

Figure-1: Landscapes of East KarbiAnglong wildlife sanctuary: a. Waterlogged area during floods. b. Peripheral zone of the sanctuary. c. River flowing through the sanctuary. d. Freshly cleared forestland for Jhum cultivation 
Table 1: Family-wise, list of plants with their habit, accession number (Ac. No.) and threat status (TS)

\begin{tabular}{l}
\hline Scientific name \\
\hline Acanthaceae \\
Lepidagathis incurva Buch.-Ham. ex D. Don \\
Phlogacanthus curviflorus (Wall.) Nees \\
Phlogacanthus parviflorus T. Anderson \\
Phlogacanthus thyrsiflorus Nees \\
Strobilanthes cusia (Nees) Kuntze \\
Thunbergia grandiflora Roxb. \\
Achariaceae \\
Hydnocarpus kurzii (King) Warb. \\
Actinidiaceae \\
Saurauia armata Kurz \\
Saurauia punduana Wall. \\
Amaranthaceae \\
Alternanthera philoxeroides (Mart.) Griseb. \\
Alternanthera sessilis (L.) R. Br. ex DC. \\
Amaranthus spinosus L. \\
Anacardiaceae \\
Rhus chinensis Mill. \\
Rhus succedanea L. \\
Annonaceae \\
Annona reticulata L.
\end{tabular}

Annona reticulata $\mathrm{L}$.

Annona squamosa L.

Goniothalamus sesquipedalis (Wall.) H.\& T

Apiaceae

Centella asiatica (L.) Urb.

Eryngium foetidum L.

Oenanthe javanica (Blume) DC.

Apocynaceae

Alstonia scholaris (L.) R. Br.

Holarrhena pubescens Wall. ex G.Don

Hoya parasitica Wall. ex Wight

Ichnocarpus frutescens (L.) W. T. Aiton

Tabernaemontana divaricata (L.) R. Br. ex

Roem. \& Schult

Wrightia coccinea (Roxb. ex Hornem.) Sims T

Araceae

Aglaonema hookerianum Schott

Alocasia macrorrhizos (L.) G. Don

Amorphophallus bulbifer (Roxb.) Blume

Homalomena aromatica (Spreng.) Schott

Pistia stratiotes L.

Rhaphidophora calophylla Schott

Sauromatum meghalayense Roy et al.

Araliaceae

Aralia armata (Wall. ex G.Don) Seem.

Brassaiopsis hainla (Buch.-Ham.) Seem.

Hydrocotyle sibthorpioides Lam.

Trevesia palmata (Roxb. ex Lindl.) Vis.

Arecaceae

Calamus tenuis Roxb.

Calamus erectus Roxb.

Caryota urens L.

Aristolochiaceae

Aristolochia indica L.

Asparagaceae

Dracaena angustifolia (Medik.) Roxb.

Balsaminaceae

Impatiens arguta Hook.f. \& Thomson

Impatiens balsamina L.

Impatiens laevigata var. laviegataHook.f.

Begoniaceae

Begonia roxburghii A.DC.

Bignoniaceae

Oroxylum indicum( L.) Kurz

Bixaceae

Bixa orellana L.

Ambient Science (2019)
Boraginaceae

Ehretia acuminata R.Br.

Calophyllaceae

Mesua ferrea L.

Trema orientalis (L.) Blume

Capparaceae

Capparis pachyphylla M.Jacobs

Caryophyllaceae

Drymaria cordata (L.) Willd. ex Schult.

Celastraceae

Microtropis discolor (Wall.) Arn.

Chloranthaceae

Chloranthus elatior Link

Clusiaceae

Garcinia pedunculata Roxb. ex Buch.-Ham. T

Combretaceae

Terminalia arjuna(Roxb. ex DC.) Wight \&Arn. T

Terminalia bellirica (Gaertn.) Roxb.

Commelinaceae

Commelina benghalensis L.

Cyanotis axillaris (L.) D.Don ex Sweet

Compositae

Ageratina adenophora (Spreng.) King \& Rob H

Ageratum conyzoides (L.) L.

Bidens pilosa $\mathrm{L}$.

Blumea fistulosa (Roxb.) Kurz

Blumea hieracifolia Hayata

Conyza japonica (Thunb.) Less. ex Less.

Convolvulaceae

Ipomoea hederifolia L.

Merremia umbellata (L.) Hallier

Costaceae

Cheilocostus speciosus (Koenig) Specht

Cucurbitaceae

Hodgsonia macrocarpa (Blume) Cogn.

Cyperaceae

Cyperus aster (Clarke ex Cherm.) Kük.

Cyperus diffusus Vahl

Cyperus rotundus L.

Fimbristylis aestivalis Vahl

Fimbristylis bisumbellata (Forssk.) Bubani

Lipocarpha chinensis (Osbeck) J.Kern

Dilleniaceae

Dillenia indica L.

Dioscoreaceae

Dioscorea alata L.

Elaeocarpaceae

Elaeocarpus lanceifolius Roxb.

Euphorbiaceae

Acalypha indica L.

Croton caudatus Geiseler

Jatropha gossypiifolia L.

Mallotus philippensis (Lam.) Müll.Arg.

Fabaceae

Abrus precatorius L.

Acacia pennata (L.) Willd.

Adenanthera pavonina L.

Bauhinia racemosa Lam.

Bauhinia variegata L.

Caesalpinia bonduc (L.) Roxb.

Callicarpa arborea Roxb.

Cassia fistula L.

Codariocalyx gyroides (Link) Hassk.

Dalbergia rimosa Roxb.

Dalbergia sissoo Roxb. ex DC.

Dalbergia stipulacea Roxb.

Desmodium elegans DC.

Entada rheedii Spreng.

Erythrina stricta Roxb.

Gymnocladus assamicus P.C.Kanjilal
$\mathrm{T} \quad \mathrm{NEHU}-12181 \quad \mathrm{NE}$

T NEHU-12331 NE

$\mathrm{T}$ NEHU-1218o NE

S NEHU-12361 E

H NEHU-12179 NE

T NEHU-12272 NE

S NEHU-12348 NE

NEHU-12178 NE

NEHU-12177 NE NEHU-12294 NE

H NEHU-12296 NE

$\mathrm{H}$ NEHU-12176 NE

NEHU-12242 NE NEHU-12243 NE NEHU-12208 NE NEHU-12307 NE NEHU-12304 NE

$\mathrm{H}$ NEHU-12309 NE

S NEHU-12267 NE

$\mathrm{H}$ NEHU-12195 NE

$\mathrm{H}$ NEHU-12175 NE

C NEHU-12332 NE

$\mathrm{H}$ NEHU-12225 NE

$\mathrm{H}$ NEHU-12229 LC

$\mathrm{H}$ NEHU-12230 NE

$\mathrm{H}$ NEHU-12238 NE

$\mathrm{H}$ NEHU-12237 LC

$\mathrm{H}$ NEHU-12224 LC

T NEHU-12221 NE

S NEHU-12174 NE

T NEHU-1226o NE

NEHU-12211 NE

$\mathrm{T}$ NEHU-1236o NE

S NEHU-12196 NE

$\mathrm{T}$ NEHU-12273 NE

S NEHU-12313 NE

$T$ NEHU-12148 NE

$\mathrm{T}$ NEHU-12330 NE

T NEHU-12315 NE

$\mathrm{T}$ NEHU-12147 LC

S NEHU-12336 NE

T NEHU-12207 NE

$T$ NEHU-12146 LC

S NEHU-12320 NE

T NEHU-12321 LC

T NEHU-12295 NE

$T$ NEHU-12285 NE

$\mathrm{S}$ NEHU-12145 LC

C NEHU-12333 NE

T NEHU-12144 NE

$\mathrm{T}$ NEHU-12253 CE

Vol.-06(2):online 
Indigofera tinctoria $\mathrm{L}$.

Parkia timoriana (DC.) Merr.

Pueraria montana (Lour.) Merr.

Senna alexandrina Mill.

Senna siamea (Lam.) H.S. Irwin \&Barneby

Senna alata (L.) Roxb.

Tadehagi triquetrum (L.) H.Ohashi

Tephrosia candida (Roxb.) DC.

Tephrosia purpurea (L.) Pers.

Fagaceae

Castanopsis indica (Roxb. ex Lindl.) A.DC.

Castanopsis tribuloides (Sm.) A.DC.

Gesneriaceae

Aeschynanthus parviflorus (D.Don) Spreng. S

Rhynchotechum ellipticum (Wall. ex Dietr.) A. DC. S

Hypoxidaceae

Molineria capitulata (Lour.) Herb.

Juglandaceae

Engelhardia spicata Lesch. ex Blume

Lamiaceae

Clerodendrum cordatum D.Don

Clerodendrum glandulosum Lindl.

Clerodendrum infortunatum $\mathrm{L}$.

Clerodendrum paniculatum $\mathrm{L}$.

Clinopodium umbrosum (M.Bieb.) Kuntze $\mathrm{H}$

Gomphostemma parviflorum Wall. ex Benth. S

Leucas aspera (Willd.) Link

Ocimum gratissimum L.

Pseudocaryopteris foetida (Don) Cantino

Lauraceae

Litsea monopetala (Roxb.) Pers.

Lecythidaceae

Careya arborea Roxb.

Lentibulariaceae

Utricularia gibba L.

Linderniaceae

Lindernia crustacea (L.) F.Muell.

Lindernia ruellioides (Colsm.) Pennell

Lythraceae

Duabanga grandiflora (DC.) Walp.

Lagerstroemia parviflora Roxb.

Lagerstroemia speciosa (L.) Pers.

Rotala rotundifolia (Buch.-Ham. ex Roxb.) Koehne

Magnoliaceae

Magnolia hodgsonii (Hook.f. \& Thomson) Keng

Magnolia insignis Wall.

Magnolia pterocarpa Roxb.

Malpighiaceae

Hiptage benghalensis (L.) Kurz

Malvaceae

Abroma augusta (L.) L.f.

Abutilon indicum (L.) Sweet

Bombax ceiba L.

Grewia serrulata DC.

Hibiscus cannabinus L.

Hibiscus sabdariffa L.

Sida rhombifolia L.

Sida acuta Burm.f.

Sterculia villosa Roxb.

Sterculiaha miltonii (Kuntze) Adelb.

Marantaceae

Phrynium pubinerve Blume

Melastomataceae

Melastoma malabathricum L.

Meliaceae

Azadirachta indica A. Juss.

Chisocheton cumingianus (C.DC.) Harms

Menispermaceae

Cissampelos pareira L.
NEHU-12143 NE

NEHU-12142 NE

NEHU-12345 NE

NEHU-12141 NE

NEHU-12140 NE

NEHU-12316 NE

NEHU-12297 NE

NEHU-12252 NE

NEHU-12289 NE

NEHU-12259 NE

NEHU-12305 NE

NEHU-12250 NE NEHU-12353 NE

H NEHU-12266 NE

T NEHU-12210 LC

S NEHU-12218 NE

S NEHU-12299 NE

S NEHU-12139 NE

NEHU-12206 NE

H NEHU-12138 NE

NEHU-12261 NE

$\mathrm{H}$ NEHU-12137 NE

$S$ NEHU-12136 NE

S NEHU-12135 NE

T NEHU-12319 NE

T NEHU-12366 NE

H NEHU-12235 LC

H NEHU-12344 LC

$\mathrm{H}$ NEHU-12351 LC

T NEHU-12200 NE

$\mathrm{T}$ NEHU-12322 NE

$\mathrm{T}$ NEHU-12220 NE

S NEHU-12343 NE

$T$ NEHU-12133 LC

T NEHU-12356 LC

$\mathrm{T}$ NEHU-12357 DD

$\mathrm{S} \quad \mathrm{NEHU}-12363 \mathrm{NE}$

S NEHU-12288 NE

$S \quad N E H U-12298 \mathrm{NE}$

$T$ NEHU-12132 NE

$\mathrm{T}$ NEHU-12302 NE

$S$ NEHU-12131 NE

$S \quad N E H U-12130$ NE

$\mathrm{S}$ NEHU-12202 NE

$S$ NEHU-12129 NE

$\mathrm{T}$ NEHU-12128 NE

T NEHU-12311 NE

H NEHU-12127 NE

S NEHU-12201 NE

T NEHU-12204 NE

T NEHU-12258 NE

$\mathrm{H} \quad \mathrm{NEHU}-12365 \mathrm{NE}$
Stephania glandulifera Miers

Menyanthaceae

Nymphoides indica (L.) Kuntze

Moraceae

Artocarpus chama Buch.-Ham.

Artocarpus heterophyllus Lam.

Ficus benghalensis $\mathrm{L}$.

Ficus hispida L.f.

Ficus racemosa L.

Streblus asper Lour.

Musaceae

Musa balbisiana Colla.

Musa acuminata Colla.

Myrtaceae

Syzygium formosum (Wall.) Masam.

Syzygium cumini (L.) Skeels

Nyctaginaceae

Boerhavia repens L.

Olacaceae

Erythropalum scandens Blume

Olax acuminata Wall. ex Benth.

Oleaceae

Nyctanthes arbor-tristis L.

Onagraceae

Oenothera rosea L'Hér. ex Aiton

Orchidaceae

Acampe praemorsa (Roxb.) Blatt. \& McCann H

Cleisostoma subulatum Blume

Dendrobium lituiflorum Lindl.

Dendrobium nobile Lindl.

Dienia ophrydis (J.König) Seidenf.

Geodorum densiflorum (Lam.) Schltr.

Habenaria dentata (Sw.) Schltr.

Hetaeria affinis (Griff.) Seidenf. \& Ormerod

Vanilla borneensis Rolfe

Oxalidaceae

Averrhoa carambola L.

Oxalis corniculata $\mathrm{L}$.

Passifloraceae

Passiflora foetida L.

Pedaliaceae

Sesamum indicum L.

Phyllanthaceae

Actephila excelsa (Dalzell) Müll. Arg.

Baccaurea ramiflora Lour.

Bridelia retusa (L.) A. Juss.

Bridelia tomentosa Blume

Phyllanthus glaucus Wall. exMüll.Arg.

Phyllanthus acidus (L.) Skeels

Phyllanthus emblica L.

Sauropus androgynus (L.) Merr.

Piperaceae

Piper nigrum L.

Piper thomsonii (C. DC.) Hook. f.

Plantaginaceae

Plantago major L.

Poaceae

Arthraxon lancifolius (Trin.) Hochst.

Bambusa affinis Munro

Bambusa pallida Munro

Bambusa tulda Roxb.

Chrysopogon aciculatus (Retz.) Trin.

Chrysopogon gryllus (L.) Trin.

Coix lacryma-jobi L.

Dendrocalamus hamiltonii Nees\&Arn. ex Munro

Dendrocalamus strictus (Roxb.) Nees

Eleusine indica (L.) Gaertn.

Eragrostis gangetica (Roxb.) Steud.

Ischaemum barbatum Retz
$\mathrm{H} \quad \mathrm{NEHU}-12265 \mathrm{NE}$

H NEHU-12231 LC

T NEHU-12126 NE

$\mathrm{T}$ NEHU-12123 NE

T NEHU-12122 NE

$\mathrm{T}$ NEHU-12121 NE

T NEHU-1212O NE

$\mathrm{T}$ NEHU-12223 NE

H NEHU-12119 NE

$\mathrm{H} \quad \mathrm{NEHU}-12118$ NE

T NEHU-12282 NE

$\mathrm{T}$ NEHU-12124 NE

$\mathrm{H} \quad \mathrm{NEHU}-12203 \mathrm{NE}$

S NEHU-12125 NE

S NEHU-12117 NE

$\mathrm{T}$ NEHU-12115 NE

$\mathrm{H}$ NEHU-12240 NE

NEHU-12116 NE NEHU-12268 NE

NEHU-12114 NE

NEHU-12310 NE

NEHU-12324 NE

NEHU-12306 NE

NEHU-12276 NE

NEHU-12227 NE

NEHU-12264 NE

T NEHU-12113 NE

$\mathrm{H}$ NEHU-12112 NE

H NEHU-12317 NE

S NEHU-12111 NE

S NEHU-12359 NE

T NEHU-12315 NE

$T$ NEHU-12209 NE

$T$ NEHU-12110 NE

$T$ NEHU-12335 NE

$T$ NEHU-12109 NE

$\mathrm{T}$ NEHU-12108 NE

$\mathrm{T}$ NEHU-12107 NE

H NEHU-12106 NE

$\mathrm{H}$ NEHU-12105 NE

H NEHU-12338 NE

$\mathrm{H} \quad \mathrm{NEHU}-12246 \mathrm{NE}$

$S \quad N E H U-12364$ NE

$\mathrm{S}$ NEHU-12104 NE

$\mathrm{S}$ NEHU-12222 NE

$\mathrm{H}$ NEHU-12341 NE

$\mathrm{H}$ NEHU-12239 NE

$\mathrm{H}$ NEHU-12339 NE

$\mathrm{S}$ NEHU-12103 NE

$\mathrm{S}$ NEHU-12102 NE

$\mathrm{H}$ NEHU-12342 NE

$\mathrm{H}$ NEHU-12236 NE

$\mathrm{H}$ NEHU-12244 NE 


\section{AMBIENT APPRAISAL}

Paspalum conjugatum P.J.Bergius

Phalaris arundinacea $\mathrm{L}$.

Polygonaceae

Persicaria glabra (Willd.) M.Gómez

Polygonum microcephalum D. Don

Pontederiaceae

Eichhornia crassipes (Mart.) Solms

Potamogetonaceae

Potamogeton alpinus Balb.

Primulaceae

Ardisia paniculata Roxb.

Rhamnaceae

Ziziphus xylopyrus (Retz.) Willd.

Rubiaceae

Catunaregam spinosa (Thunb.) Tirveng.

Chassalia curviflora (Wall.) Thwaites

Geophila repens (L.) I. M. Johnst.

Ixora polyantha Wight

Mussaenda roxburghii Hook. f.

Ophiorrhiza mungos L.

Paederia foetida $\mathrm{L}$.

Psychotria calocarpa Kurz

Psychotria montana Blume

Rutaceae

Citrus assamensis Dutta \& Bhattacharya

Micromelum pubescens Blume

Murraya koenigii (L.) Spreng.

Zanthoxylum rhetsa (Roxb.) DC.

Sapindaceae

Aesculus assamica Griff.

Lepisanthes senegalensis (Poir.) Leenh.

Saururaceae

Houttuynia cordata Thunb.

Simaroubaceae

Brucea mollis Wall. ex Kurz

Picrasma javanica Blume

Smilacaceae

Smilax ovalifolia Roxb. ex D. Don

Solanaceae

Physalis peruviana L.

Physalis angulata $\mathrm{L}$.

Solanum spirale Roxb.

Stemonaceae

Stemona tuberosa Lour.

Theaceae

Camellia caudata Wall.

Schima wallichii Choisy

Urticaceae

Oreocnide integrifolia (Gaudich.) Miq.

Sarcochlamys pulcherrima Gaudich.

Verbenaceae

Lippia alba (Mill.) Br. ex Britton \& Wilson

Vitaceae

Leea asiatica (L.) Ridsdale

Cayratia pedate (Wall.) Gagnep.

Leea indica (Burm. f.) Merr.

Zingiberaceae

Alpinia nigra (Gaertn.) Burtt

Boesenbergia longiflora (Wall.) Kuntze

Curcuma aromatica Salisb.

Globba multiflora Wall. ex Baker

Hedychium coronarium J. Koenig

Larsenianthus assamensis Dey, Mood \& Choudhury

Larsenianthus careyanus (Benth.) Kress \& Mood

Zingiber chrysanthum Roscoe

Zingiber rubens Roxb.

(Abbreviations: $\mathrm{H}=$ Herb, $\mathrm{S}$ = Shrub, T = Tree, C = Climber; IUCN

status: $\mathrm{E}=$ Endangered, $\mathrm{CE}=$ Critically Endangered, $\mathrm{V}=$ Vulnerable, $\mathrm{DD}=$ Data Deficient, LC = Least Concern and NE = Not Evaluated)

H NEHU-12245 LC

$\mathrm{H}$ NEHU-12340 LC

$\mathrm{H} \quad \mathrm{NEHU}-12226 \mathrm{NE}$

$\mathrm{H}$ NEHU-12101 NE

H NEHU-12234 NE

H NEHU-12228 LC

S NEHU-12271 NE

T NEHU-1210o NE

T NEHU-12286 NE

S NEHU-12099 NE

$\mathrm{H} \quad \mathrm{NEHU}-12098 \mathrm{NE}$

NEHU-12263 NE

NEHU-12199 NE

NEHU-12354 NE

NEHU-12317 NE

NEHU-12274 NE

NEHU-12277 NE

NEHU-12023 NE NEHU-12219 NE

NEHU-12097 NE

NEHU-12077 NE

NEHU-12325 NE

NEHU-12287 NE

H NEHU-12257 NE

T NEHU-12215 T

T NEHU-12352 NE

S NEHU-12350 NE

$\mathrm{H} \quad \mathrm{NEHU}-12065 \mathrm{NE}$

$\mathrm{H}$ NEHU-12280 NE

S NEHU-12318 NE

H NEHU-12066 NE

T NEHU-12248 LC

T NEHU-12314 NE

T NEHU-12056 NE

$S$ NEHU-12212 NE

S NEHU-12059 NE

NEHU-12256 NE

NEHU-12049 V

NEHU-12367 NE

$\mathrm{H} \quad \mathrm{NEHU}-12048 \mathrm{NE}$

$\mathrm{H} \quad \mathrm{NEHU}-12262 \mathrm{NE}$

$\mathrm{H}$ NEHU-12300 NE

$\mathrm{H} \quad \mathrm{NEHU}-12254 \mathrm{NE}$

$\mathrm{H}$ NEHU-12301 NE

NEHU-12047 NE

NEHU-12251 NE

NEHU-12197 NE

NEHU-12046 NE

Ambient Science (2019)

http://www.caves.res.in/

Ambient Science, 2019: Vol. 06(2); Online DOI:10.21276/ambi.2019.06.2.aa03

Table 2: Population of Threatened taxa within EKWLS

Species and Vegetation type (Champion \& Seth, 1968)

Mature Saplings Seedling Total Threat status (Reference)

Brucea mollis in: Moist semi-evergreen forests

$27 \quad 12 \quad 10 \quad 49 \quad$ E (Borthakur et. al., 2018)

Capparis pachyphylla in: Moist mixed deciduous forests

$$
50 \quad 0 \quad 0 \quad 5 \quad \text { E (IUCN) }
$$

Cayratia pedata in: Moist semi-evergreen forests

$$
\begin{array}{lllll}
7 & 0 & 0 & 7 & \text { V (IUCN) }
\end{array}
$$

Gymnocladus assamicus in: Moist mixed deciduous forests
9
13 CE (IUCN)

Hydnocarpus kurzii in: Moist semi-evergreen forests
$21 \quad 10$
15
46 T (Majumdar et al., 2019 )

Saurauia punduana in: Moist semi-evergreen forests

$$
\begin{array}{lllll}
7 & 2 & 0 & 9 & \text { CE (IUCN) }
\end{array}
$$

Vanilla borneensis in: Moist semi-evergreen forests

$$
\begin{array}{lllll}
2 & 1 & 0 & 3 & \mathrm{~T} \text { (Deka et al., 2017) }
\end{array}
$$

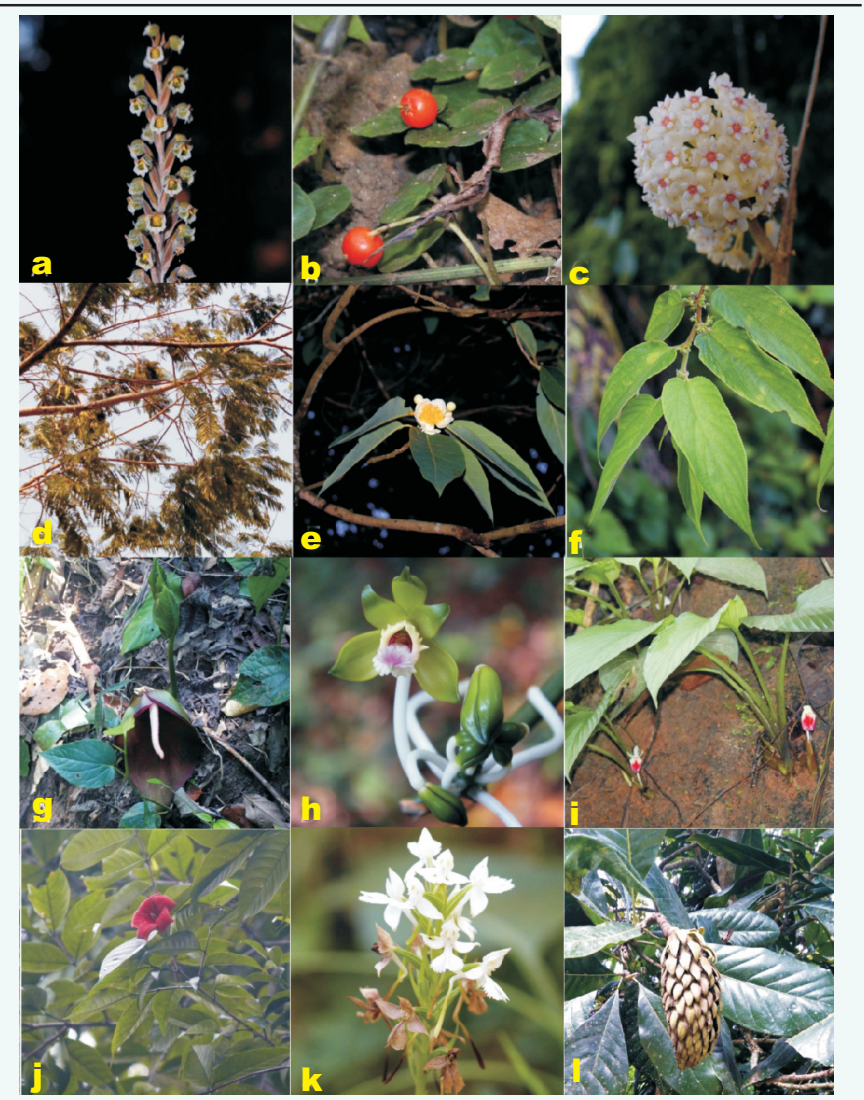

Figure-2: a) Hetaeria affinis b) Geophila repens c) Hoya parasitica d) Gymnocladus assamicus e) Schima wallichii f) Trema orientalis g) Sauromatum meghalayense h) Vanilla borneensis i) Boesenbergia longiflora j) Wrightia coccinea k) Habenaria dentata 1) Magnolia pterocarpa

Mood \& Choudhury, Globba multiflora Wall. ex Baker and Citrus assamensis Dutta \& Bhattacharya. The work also states a new distributional record of Sauromatum meghalayense to the state of Assam was first reported from the state of Meghalaya (Talukdar et al., 2014) (Fig.-1,2).

Threat categories: of all the species, 4 species were in threatened catagories according to IUCN 2019, which 
includes 2 Critically Endangered (Gymnocladus assamicus, Saurauia punduana), one Endangered (Capparis pachyphylla), 1 Vulnerable (Cayratia pedata) and 3 species were listed as regionally threatened by different literatures (Brucea mollis, Hydnocarpus kurzii, Vanilla borneensis) and the other species are categorized as Data Deficient, Least Concern or are within Not Evaluated categories.

Population and regeneration status of the threatened species: Of the threatened species population of Brucea mollis was highest with 27 adults Individuals, followed by Hydnocarpus kurzii (21), Gymnocladus assamicus (9), Cayratia pedata (7), Saurauia punduana (7), Capparis pachyphylla (5) and Vanilla borneensis (2). Among them Brucea mollis and Hydnocarpus kurzii has shown a high regeneration, whereas Capparis pachyphylla, Cayratia pedate and Vanilla borneensis with meager regeneration rates, based on number of seedling and sapling present in the study site (Table-2).

\section{Conclusion:}

EKAWLS is covered on almost all the sides by human habitation, and due to a clearing of lands for Jhum practice, collection of firewood and non-timber forest products, has put the present vegetation under severe threat. Therefore there is an urgent need for effective conservation stretegies to safeguard the existing resources. Moreover, the population of those threatened species needs to protect strictly and monitored within its natural habitat. Illegal timber extraction, shifting cultivation, forest fires, and illicit agricultural expansion, need to be check-in and around the population of this species. Moreover, there are no known reports on the diversity of plants within the region, hence this report will serve as a baseline for future considerations.

\section{Acknowledgments:}

The first author is thankful to the University Grant Commission (UGC), New Delhi for the financial support through National Fellowship for Higher Education of ST students. We also extend our sincere thanks to the PCCF, Karbi Anglong Division \& Forest Department of Assam for granting permission to carry out this study. Authors are also grateful to the Head, Centre for Advanced Studies in Botany, NEHU Shillong for providing necessary facilities and Botanical Survey of India (Eastern Circle, Shillong) for consultation the library and herbaria.

\section{References:}

Bora, A. \& Bhattacharyya, D. (2017): Phytodiversity of Barail Wildlife Sanctuary, Assam, India: field-based observations-I. Trees and lianas. Check List. 13(6):1037-1053.

Borthakur, S.K., Baruah, P.S., Deka, K., Das, P., Sarma, B., Adhikari, D. \& Tanti, B. (2018): Habitat distribution modelling for improving conservation status of Brucea mollis Wall. ex Kurz.-an endangered potential medicinal plant of Northeast India. L. Nat. Conserv., 42:104-110.

Deka, K., Baruah, P.S., Sarma, B., Borthakur, S.K. \& Tanti, B. (2017): Preventing extinction and improving conservation status of Vanilla borneensis Rolfe- A rare, endemic and threatened orchid of Assam, India.J. Nat. Conserv.,37:39-46.

Gogoi, K. \& Yonzone, R. (2013): Orchids of the Karbi Anglong District of Assam, India.MOIS J., 14(4):1-20.

Hansen, M.C., Potapov, P.V., Moore, R., Hancher, M., Turubanova, S.A., Tyukavina, A.L., Justice, C.O. \& Townshend, J.R.G. (2013): High-resolution global maps of 21st-century forest cover change. Science, 342(6160):850-853.

Jain, S.K. \& Rao, R.R. (1977): Handbook of Field and Herbarium Methods. Pub. by: Today and Tomorrow's Printers and Publishers, New Delhi, India. P. 157.

Kar, A. \& Borthakur, S.K. (2008): Wild Vegetation of Karbi Anglong District, Assam. Nat. Prod. Radiance, 7(5):448-46o.

Majumdar, K., Adhikari, D., Datta, B.K. \& Barik, S.K. (2019): Identifying corridors for landscape connectivity using species distribution modeling of Hydnocarpus kurzii (King) Warb., a threatened species of the Indo-Burma Biodiversity Hotspot. Landsc. Ecol. Eng., 15(1):13-23.

Mir, A.H., \& Upadhaya, K. (2017): Effect of traditional management practices on woody species composition and structure in montane subtropical forests of Meghalaya, Northeast India. L. Mountain Sci., 14(8):1500-1512.

Mir, A.H., Upadhaya, K., Odyuo, N. \&Tiwari, B.K. (2017a): Rediscovery of Magnolia rabaniana (Magnoliaceae): A threatened tree species of Meghalaya, northeast India. J. Asia Pac. Biodiv., 10(1):127-131.

Mir, A.H., Upadhaya, K. \& Roy, D.K. (2017b): Rediscovery, distribution and conservation implications of Cleyera grandiflora Wall. ex Choisy (Pentaphylacaceae): an endangered and endemic tree species of Meghalaya, Northeast India. Nat. Acad. Sci. Lett., 40(3):205-209.

Phongchopi, U., Tamuli, A.K. \& Teron, R. (2014): Inventory of wild food plants in Marat Longri Wildlife Sanctuary in Assam, India. Pleione. 8(2):331-343.

Phongchopi, U., Teron, R. \& Tamuli, A.K. (2017): Conservation Crisis in Marat Longri Wildlife Sanctuary, Assam, India. Ambient Sci., 04(1):67-73.

Ronghang, R., Teron, R., Tamuli, A.K. \& Rajkhowa, C.R. (2012): Tribal societies and deforestation in Karbi Anglong District of Assam (India). Ecoscan, 1:231-236.

Sankar, S. \& Chowdhury, S. (2000): Survey of diversity of living economic flora and their conservation and development in the Karbi Anglong District of Assam. Envis Bull., 8:1-4.

Talukdar, A.D., Roy, D.K., Sinha, B.K. \& Choudhury, M.D. (2014): Sauromatum meghalayense (Araceae; Tribe: Areae), a new species from Meghalaya, India. $\underline{\mathrm{NeBIO}}$. 5(3):1-3.

Teron, R. \& Borthakur, S.K. (2009): Traditional knowledge relating to use of flora and fauna as indicators in predicting annual seasons among Karbi tribe of Assam. Indian J. Trad. Know. $8(4): 518-524$.

Teron, R. \& Borthakur, S.K. (2012): Traditional knowledge of herbal dyes and cultural Significance of colors among the Karbis Ethnic Tribe in Northeast India. Ethnobot. Res. Appl., 10:593-604.

Teron, R. \& Borthakur, S.K. (2013): Folklore claims of some medicinal plants as antidote against poisons among the Karbis of Assam. Pleione. 7(2):346-356.

Teronpi, N., Terongpi, R., Tamuli, A.K. \& Teron, R. (2015): Ethnobotanical investigations on antidotes in Singhason hills, Karbi Anglong district. Int. I. Res. Ayur. Phar., 6(1):150-156. 